\title{
Physiologic Response to the Pfizer-BioNTech COVID-19 Vaccine Measured Using Wearable Devices: Prospective Observational Study
}

Alexander G Hajduczok ${ }^{1 *}$, MD; Kara M DiJoseph ${ }^{1 *}$, DO; Brinnae Bent ${ }^{2}$, PhD; Audrey K Thorp ${ }^{1}$, DO; Jon B Mullholand $^{1}$, MD; Stuart A MacKay ${ }^{1}$, DO; Sabrina Barik ${ }^{1}$, MD; Jamie J Coleman ${ }^{3}$, MD; Catharine I Paules ${ }^{4}$, MD; Andrew Tinsley ${ }^{5}$, MD

\footnotetext{
${ }^{1}$ Division of Internal Medicine, Department of Medicine, Penn State Health Milton S. Hershey Medical Center, Hershey, PA, United States

${ }^{2}$ Department of Biomedical Engineering, Duke University, Durham, NC, United States

${ }^{3}$ Department of Surgery, Denver Health Medical Center, Denver, CO, United States

${ }^{4}$ Division of Infectious Diseases, Department of Medicine, Penn State Health Milton S. Hershey Medical Center, Hershey, PA, United States

${ }^{5}$ Division of Gastroenterology, Department of Medicine, Penn State Health Milton S. Hershey Medical Center, Hershey, PA, United States

*these authors contributed equally
}

\section{Corresponding Author:}

Alexander G Hajduczok, MD

Division of Internal Medicine

Department of Medicine

Penn State Health Milton S. Hershey Medical Center

500 University Drive

Hershey, PA, 17033

United States

Phone: 17168634928

Email: alexander.hajduczok@jefferson.edu

\section{Abstract}

Background: The Pfizer-BioNTech COVID-19 vaccine uses a novel messenger RNA technology to elicit a protective immune response. Short-term physiologic responses to the vaccine have not been studied using wearable devices.

Objective: We aim to characterize physiologic changes in response to COVID-19 vaccination in a small cohort of participants using a wearable device (WHOOP Strap 3.0). This is a proof of concept for using consumer-grade wearable devices to monitor response to COVID-19 vaccines.

Methods: In this prospective observational study, physiologic data from 19 internal medicine residents at a single institution that received both doses of the Pfizer-BioNTech COVID-19 vaccine was collected using the WHOOP Strap 3.0. The primary outcomes were percent change from baseline in heart rate variability (HRV), resting heart rate (RHR), and respiratory rate (RR). Secondary outcomes were percent change from baseline in total, rapid eye movement, and deep sleep. Exploratory outcomes included local and systemic reactogenicity following each dose and prophylactic analgesic use.

Results: In 19 individuals (mean age 28.8, SD 2.2 years; n=10, 53\% female), HRV was decreased on day 1 following administration of the first vaccine dose (mean $-13.44 \%$, SD 13.62\%) and second vaccine dose (mean $-9.25 \%$, SD 22.6\%). RHR and RR showed no change from baseline after either vaccine dose. Sleep duration was increased up to 4 days post vaccination, after an initial decrease on day 1. Increased sleep duration prior to vaccination was associated with a greater change in HRV. Local and systemic reactogenicity was more severe after dose two.

Conclusions: This is the first observational study of the physiologic response to any of the novel COVID-19 vaccines as measured using wearable devices. Using this relatively small healthy cohort, we provide evidence that HRV decreases in response to both vaccine doses, with no significant changes in RHR or RR. Sleep duration initially decreased following each dose with a subsequent increase thereafter. Future studies with a larger sample size and comparison to other inflammatory and immune biomarkers such as antibody response will be needed to determine the true utility of this type of continuous wearable monitoring in regards to vaccine responses. Our data raises the possibility that increased sleep prior to vaccination may impact physiologic 
responses and may be a modifiable way to increase vaccine response. These results may inform future studies using wearables for monitoring vaccine responses.

Trial Registration: ClinicalTrials.gov NCT04304703; https://www.clinicaltrials.gov/ct2/show/NCT04304703

(JMIR Form Res 2021;5(8):e28568) doi: 10.2196/28568

\section{KEYWORDS}

COVID-19; wearable devices; remote physiologic monitoring; heart rate; heart rate variability; respiratory rate; sleep; REM sleep; deep sleep; wearable; vaccine; monitoring; respiratory; physiological; cohort

\section{Introduction}

The COVID-19 pandemic has had a substantial global impact resulting in over 165 million infections and nearly 3.5 million deaths worldwide [1,2]. Vaccines are required to end the pandemic. The first vaccine to receive emergency use authorization for prevention of COVID-19 infection by the United States Food and Drug Administration was the BNT162b2 messenger RNA (mRNA) COVID-19 vaccine (Pfizer-BioNTech COVID vaccine) that encodes the spike protein of the SARS-CoV-2 virus [3,4]. Following preliminary studies with this mRNA vaccine showing neutralizing antibody response, a phase 3 randomized clinical trial demonstrated that the Pfizer-BioNTech vaccine was safe and had an efficacy of $95 \%$ in reducing risk of contracting COVID-19 compared to placebo [4-6].

An estimated $21 \%$ of US adults report using wearable devices that objectively measure physiologic parameters [7]. Although marketed for personal use, the widespread nature and convenience of these devices allows health care professionals to monitor physiologic changes in real time [8]. The WHOOP Strap 3.0 has been externally validated for tracking of heart rate variability (HRV), resting heart rate (RHR), respiratory rate (RR), and sleep stage duration [9]. HRV is determined by the subtle variation in the time between successive heart beats, thus
HRV is a measure of the balance between the sympathetic and parasympathetic nervous system and their composite effects on heart rate [10].

A recent study using the WHOOP device was able to track physiologic changes, specifically an increase in nocturnal RR and decrease in HRV, in individuals who reported COVID-19 infection. These changes were noted 2 days before symptom onset in $20 \%$ of individuals and in $80 \%$ of the cohort after symptom onset [11]. Other studies have used HRV and RR measured by wearable devices to prospectively and retrospectively predict and identify COVID-19 infection (confirmed by positive testing) [12-14]. Therefore, we postulated that it would be possible to track an array of physiologic responses following COVID-19 vaccination. This wearable remote monitoring strategy could serve as a proof of concept and guide design of future studies of the physiologic and immune responses to vaccines.

\section{Methods}

\section{Study Population}

Internal medicine residents at Penn State Hershey Medical Center previously enrolled in a clinical trial (NCT04304703) using the WHOOP Strap 3.0 to measure physiologic parameters were used for this analysis (Multimedia Appendix 1, Figure 1) [15].

Figure 1. A total of 19 participants, 53\% female, who were vaccinated with two doses of the Pfizer-BioNtech COVID-19 vaccine (mean time between doses 19.6, SD 2.8 days), transmitted continuous physiologic data via the WHOOP device. Changes from baseline were observed in HRV and were most pronounced on day 1 and 2 for dose 1 and only day 1 for dose 2 . RR and RHR were unaffected following vaccination. Sleep duration initially decreased on day 1 post vaccine dose 1 and dose 2, with a compensatory increase from days 2 to 4 , prior to return to baseline. Sleep deprivation was associated with a blunted HRV response, and premedication was associated with less change in RR and increases in REM and deep sleep percentages. HRV: heart rate variability; REM: rapid eye movement; RHR: resting heart rate; RR: respiratory rate.

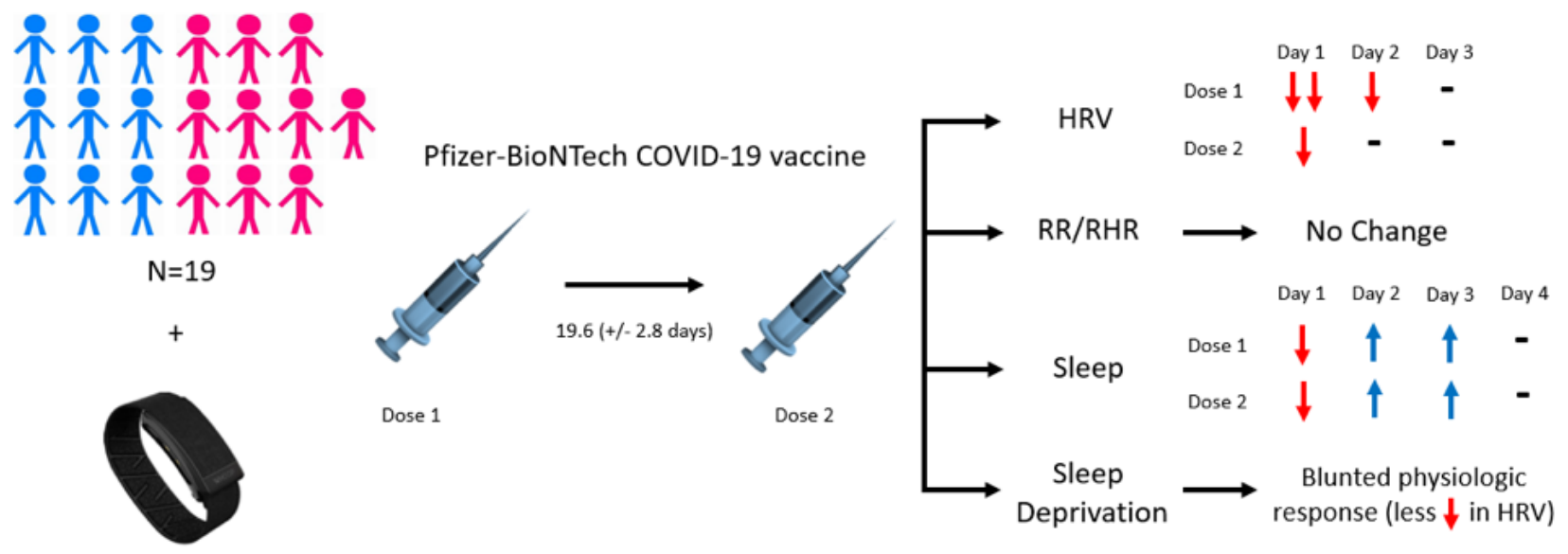




\section{Study Design}

The primary objective of this prospective observational study was to determine the physiologic changes following the first and second doses of the Pfizer-BioNTech COVID-19 vaccine. Primary outcomes were percent change from baseline in HRV, $\mathrm{RR}$, and RHR for days 1 to 6 following each vaccine dose. Secondary outcomes were percent change from baseline duration of total, rapid eye movement (REM), and deep sleep. Exploratory outcomes included analysis of local and systemic reactogenicity (type and duration) associated with vaccination and prophylactic analgesic use.

\section{Study Procedures}

Internal medicine residents were given a WHOOP Strap 3.0 to wear to measure physiologic parameters and sleep [15] (for full details, see Multimedia Appendix 2 [9,16-19]). Eligible participants were surveyed to disclose their vaccination dates for the novel Pfizer-BioNTech COVID-19 vaccine along with local and systemic reactogenicity and analgesic use following each vaccine dose.

Inclusion criteria for this analysis were patients concurrently enrolled in a clinical trial (NCT04304703) using the WHOOP device and who transmitted at least $80 \%$ of physiologic data during the study period including at least 24 of 45 days prior to dose one (to establish baseline metrics) and all data for the 6 days following vaccine dose one, dose two, or both. These data cutoffs were chosen based on published data using the WHOOP device for establishing a change from baseline in RR [11]. Local and systemic reactogenicity was graded as mild, moderate, or severe based on guidelines from the Centers for Disease Control and Prevention $[1,20]$. Patients were excluded if they did not or were unable to disclose the dates of vaccination (Multimedia Appendix 3). Data were blinded to study investigators for analysis. Recorded demographics included age, gender, comorbidities, and year of residency training.

Data collection was approved by the Institutional Board Review at Penn State Hershey Medical Center (STUDY14522).

\section{Statistical Analysis}

We defined a significant change from baseline to be greater than $5 \%$ a priori. This cutoff was set based on recent findings in two studies: (1) changes in RR and other physiologic parameters in COVID-19-positive individuals, which were used to develop a predictive algorithm for COVID-19 infection risk stratification [11], and (2) precision measurements of heart rate, RR, HRV, and REM sleep stage duration using the WHOOP device were found to have less than $10 \%$ error [9].

The percent change of each metric for each participant in the data set was averaged together for the overall percent change of that metric for each day (d; equation 1$)$. In equation $1, b_{n}$ is given as the average of the metric from the baseline period for participant $n$, and $x_{n}$ is the value of the metric on the day (d) being calculated post vaccine dose.

$$
(\text { Mean Percent Change })_{d}=\frac{\sum_{i=0}^{n}\left(\frac{b_{n}-x_{n d}}{b_{n}} \times 100 \%\right)}{n}, \quad b_{n}=\frac{\sum i_{n_{0}}}{n_{o}}
$$

To determine the effect of sleep for the week leading up to the vaccine on the physiological effects of the vaccine, we computed Pearson correlations between hours of sleep in the 7 days prior to vaccine dose 1 and the percent changes of the physiological measurements post vaccine dose 1 [21].

Symptoms were aggregated and the density of the self-reported duration of symptoms was calculated [22]. Postvaccination reaction severity was compared to changes in physiologic parameters by Pearson correlations.

\section{Results}

\section{Baseline Characteristics}

A total of 19 participants met inclusion and exclusion criteria for this analysis; 18 individuals for dose 1 and 13 for dose 2 (Multimedia Appendix 3). Participants were 53\% $(\mathrm{n}=10)$ female, with an age range of 26 to 35 years and a mean and median age of 28.8 (SD 2.2) and 29 years, respectively. No comorbidities were reported in $74 \% \quad(n=14)$ of participants (Multimedia Appendix 1).

Baseline metrics were collected for all participants up to 45 days prior to vaccination dose 1 (Table 1). Mean baselines were as follows: RHR 63.09 (SD 6.36) bpm, HRV 52.09 (SD 21.58) $\mathrm{ms}$, and RR 16.27 (SD 1.23) respirations per minute (rpm). Although interindividual variability in metrics had a wider range, intraindividual variability was much lower, most notably in nocturnal RR, with an intraindividual SD of mean 0.37 (SD $0.12) \mathrm{rpm}$.

Table 1. Baseline physiological and sleep metrics intraindividual mean and SD.

\begin{tabular}{lll}
\hline Metric & Intraindividual mean, mean (SD) & Intraindividual SD, mean (SD) \\
\hline Heart rate variability (ms) & $52.09(21.58)$ & $13.16(6.94)$ \\
Resting heart rate (bpm) & $63.09(6.36)$ & $4.95(1.50)$ \\
Respiratory rate (rpm) & $16.27(1.23)$ & $0.37(0.12)$ \\
Sleep (hours) & $6.71(0.58)$ & $1.48(0.39)$ \\
REM $^{\mathrm{a}}$ sleep (\%) & $21.99(6.71)$ & $6.49(1.23)$ \\
Deep sleep (\%) & $19.10(2.15)$ & $3.81(0.66)$ \\
\hline
\end{tabular}

${ }^{\mathrm{a}} \mathrm{REM}$ : rapid eye movement. 


\section{Physiologic Response to COVID-19 Vaccination by Dose}

For dose $1(\mathrm{n}=18)$, there was a reduction in HRV on day 1 (mean percent change $-13.44 \%$, SD $13.62 \%$ ). HRV returned to baseline by day 3 and remained at baseline thereafter (Figure 2A, blue; Table 2). There was no significant change in RHR and RR compared to baseline in the 6 days following vaccination (Figure 2B, C, blue; Table 2).

Figure 2. Percent change from baseline in (A) heart rate variability, (B) respiratory rate, and (C) resting heart rate, measured 6 days following COVID-19 vaccine dose 1 (blue) and 2 (magenta). Data is reported as mean (SD).

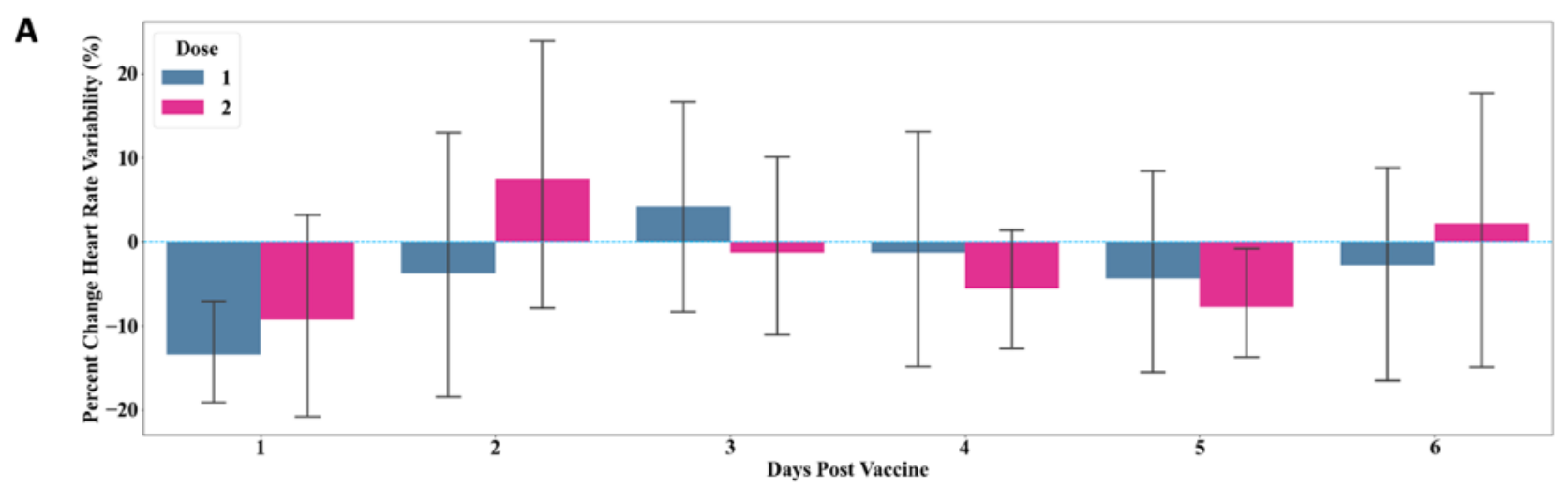

B

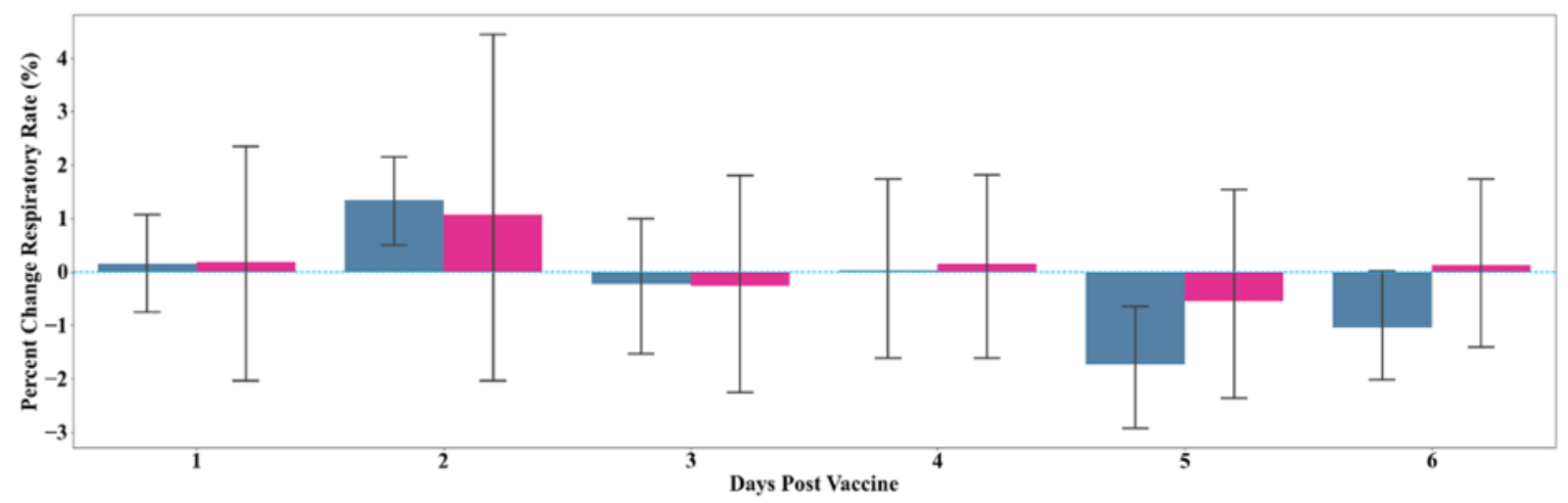

C

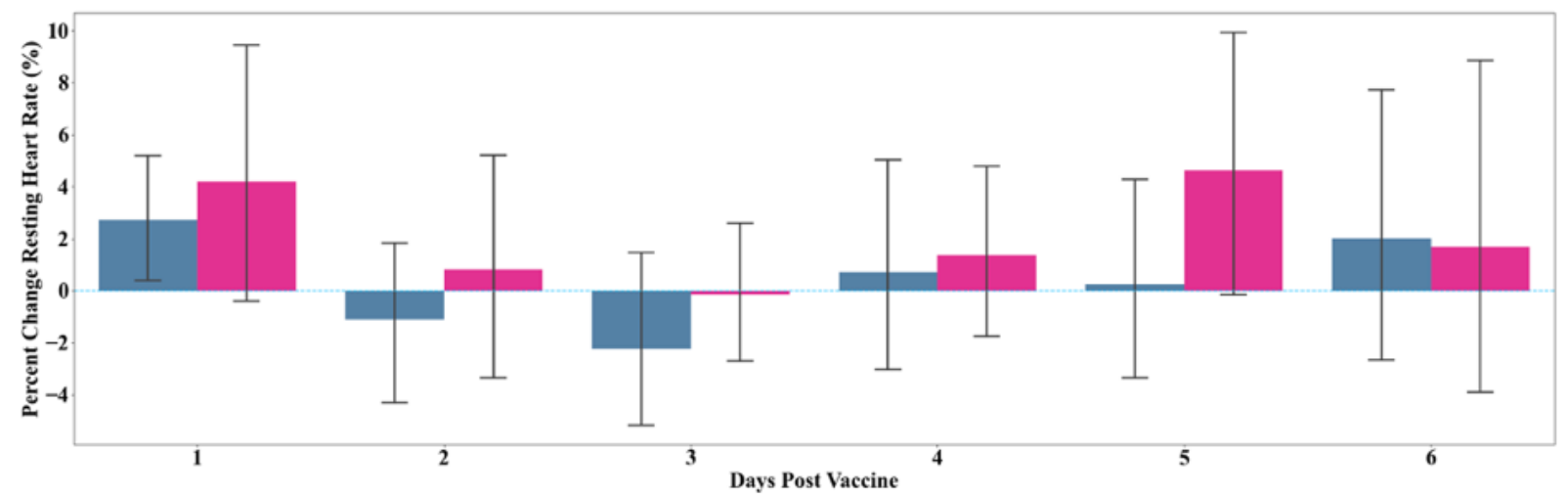

For dose $2(\mathrm{n}=13)$, HRV decreased on day 1 (mean percent change $-9.25 \%$, SD $22.69 \%$ ) but quickly normalized to baseline by day 2 (Figure 2A, magenta; Table 3). Similar to dose 1 , there was no significant change in RHR and RR in response to dose 2 , with both metrics remaining at baseline from day 1 to day 6 (Table 3). 
Table 2. Percent changes from baseline in physiological and sleep metrics for 6 days postvaccine dose $1(\mathrm{n}=18)$.

\begin{tabular}{|c|c|c|c|c|c|c|}
\hline Metrics & $\begin{array}{l}\text { Day } 1, \text { mean per- } \\
\text { cent change (SD) }\end{array}$ & $\begin{array}{l}\text { Day } 2 \text {, mean per- } \\
\text { cent change (SD) }\end{array}$ & $\begin{array}{l}\text { Day } 3 \text {, mean per- } \\
\text { cent change }(\mathrm{SD})\end{array}$ & $\begin{array}{l}\text { Day } 4, \text { mean per- } \\
\text { cent change (SD) }\end{array}$ & $\begin{array}{l}\text { Day } 5 \text {, mean per- } \\
\text { cent change (SD) }\end{array}$ & $\begin{array}{l}\text { Day } 6 \text {, mean per- } \\
\text { cent change (SD) }\end{array}$ \\
\hline Heart rate variability & $-13.44(13.62)$ & $-3.74(34.63)$ & $4.21(27.23)$ & $-1.32(30.39)$ & $-4.35(26.79)$ & $-2.80(27.46)$ \\
\hline Resting heart rate & $2.73(5.50)$ & $-1.10(6.93)$ & $-2.23(7.31)$ & $0.72(8.80)$ & $0.26(8.68)$ & $2.02(11.48)$ \\
\hline Respiratory rate & $0.16(1.95)$ & $1.34(1.98)$ & $-0.23(2.70)$ & $0.02(3.81)$ & $-1.73(2.56)$ & $-1.04(2.29)$ \\
\hline Hours of sleep & $-8.41(22.96)$ & $5.00(18.27)$ & $9.41(21.60)$ & $7.74(17.81)$ & $3.21(24.38)$ & $-3.21(27.54)$ \\
\hline Percent of REM ${ }^{\mathrm{a}}$ sleep & $-4.94(37.65)$ & $-6.53(30.06)$ & $-5.13(33.34)$ & $-6.70(19.62)$ & $-2.70(31.10)$ & $-16.98(29.41)$ \\
\hline Percent of deep sleep & $9.64(26.30)$ & $3.08(23.00)$ & $4.11(12.66)$ & $4.58(15.28)$ & $-6.05(19.58)$ & $-2.38(17.53)$ \\
\hline
\end{tabular}

${ }^{\mathrm{a}} \mathrm{REM}$ : rapid eye movement.

Table 3. Percent changes from baseline in physiological and sleep metrics for 6 days postvaccine dose $2(\mathrm{n}=13)$.

\begin{tabular}{|c|c|c|c|c|c|c|}
\hline Metrics & $\begin{array}{l}\text { Day } 1, \text { mean per- } \\
\text { cent change (SD) }\end{array}$ & $\begin{array}{l}\text { Day } 2, \text { mean per- } \\
\text { cent change (SD) }\end{array}$ & $\begin{array}{l}\text { Day } 3 \text {, mean per- } \\
\text { cent change (SD) }\end{array}$ & $\begin{array}{l}\text { Day } 4, \text { mean per- } \\
\text { cent change (SD) }\end{array}$ & $\begin{array}{l}\text { Day } 5 \text {, mean per- } \\
\text { cent change (SD) }\end{array}$ & $\begin{array}{l}\text { Day } 6, \text { mean per- } \\
\text { cent change (SD) }\end{array}$ \\
\hline Heart rate variability & $-9.25(22.69)$ & $7.48(32.44)$ & $-1.30(19.44)$ & $-5.56(13.70)$ & $-7.76(13.12)$ & $2.19(30.22)$ \\
\hline Resting heart rate & $4.20(9.42)$ & $0.82(8.27)$ & $-0.15(5.15)$ & $1.37(6.17)$ & $4.63(10.38)$ & $1.70(12.83)$ \\
\hline Respiratory rate & $0.19(4.10)$ & $1.07(6.44)$ & $-0.26(4.00)$ & $0.15(3.22)$ & $-0.54(3.63)$ & $0.13(3.06)$ \\
\hline Hours of sleep & $-2.10(26.18)$ & $5.33(17.71)$ & $6.06(23.84)$ & $9.22(28.37)$ & $-4.58(20.45)$ & $0.49(12.30)$ \\
\hline Percent of REM ${ }^{\mathrm{a}}$ sleep & $13.79(45.88)$ & $-8.73(39.57)$ & $12.67(36.41)$ & $0.64(37.99)$ & $6.12(32.02)$ & $-8.35(43.06)$ \\
\hline Percent of deep sleep & $4.00(25.02)$ & $-1.70(19.21)$ & $3.56(20.31)$ & $-6.01(22.09)$ & $3.42(21.31)$ & $-11.37(24.40)$ \\
\hline
\end{tabular}

${ }^{\mathrm{a}} \mathrm{REM}$ : rapid eye movement.

\section{Postvaccination Changes in Sleep}

Total sleep duration, REM, and deep sleep duration (in hours) were measured for all participants for 6 days following vaccine administration. Total sleep duration followed a similar overall pattern for both vaccine doses: an initial decrease was observed on day 1 (dose 1 : mean $-8.41 \%$, SD $22.96 \%$; dose 2 : mean $-2.1 \%$, SD $26.8 \%$ ) followed by an increase above baseline on days 2, 3, and 4, with subsequent return to baseline on days 5 to 6 (Figure 3A; Tables 2 and 3). The change in sleep duration peaked on day 3 following dose 1 and day 4 following dose 2 . Total sleep duration was proportional to time in bed and thus showed similar trends in response to vaccine dose 1 and dose 2 .

Patterns of change in REM and deep sleep did not follow the same pattern as total sleep duration but showed greater variability overall (Figure 3B, C; Tables 2 and 3). Total sleep cycles and sleep disturbances had no correlation with changes in physiologic metrics following either vaccine dose. 
Figure 3. Percent change from baseline in (A) total sleep duration, (B) REM sleep duration, and (C) deep sleep duration, measured 6 days following COVID-19 vaccine dose 1 (blue) and 2 (magenta). Data is reported as mean (SD). REM: rapid eye movement.

A

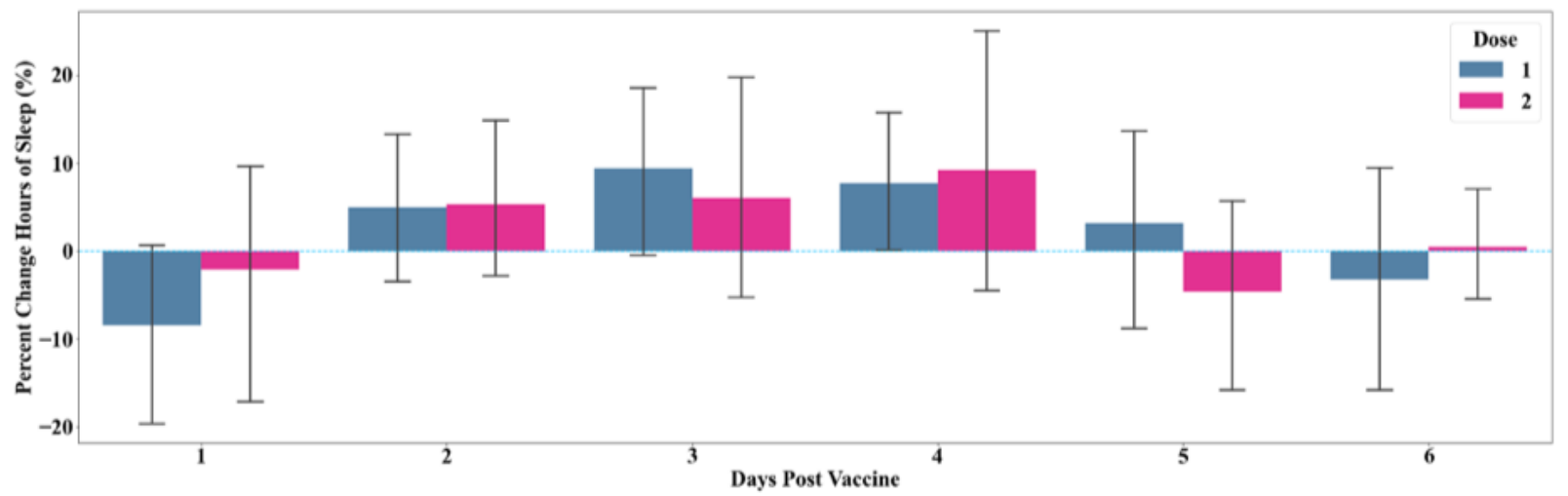

B

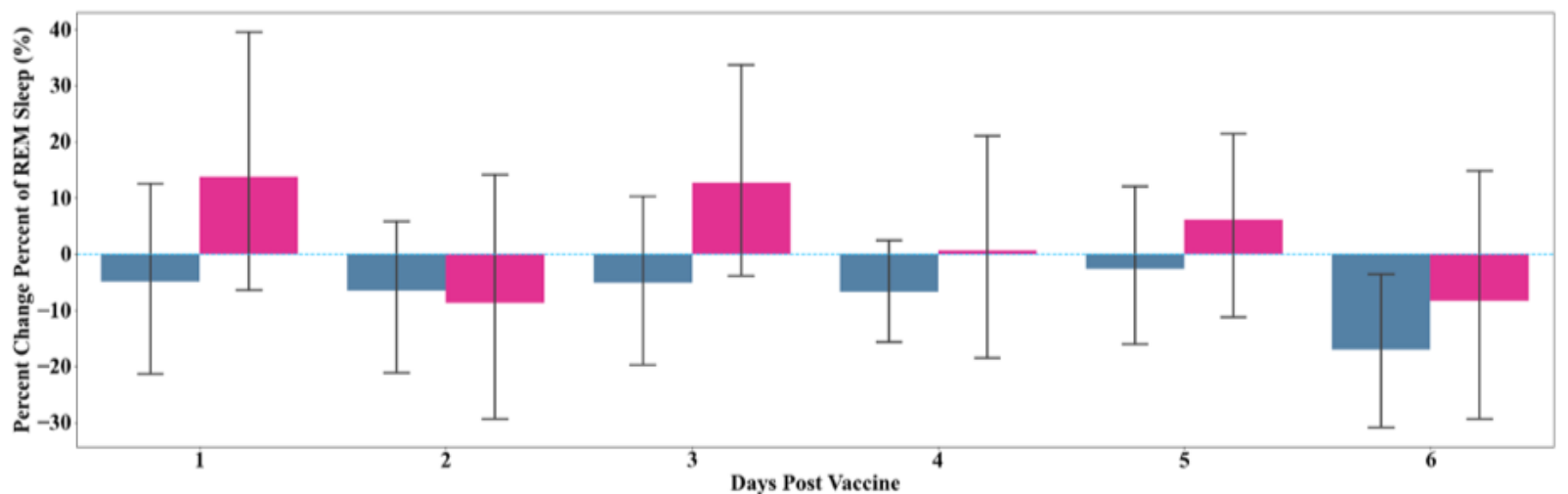

C

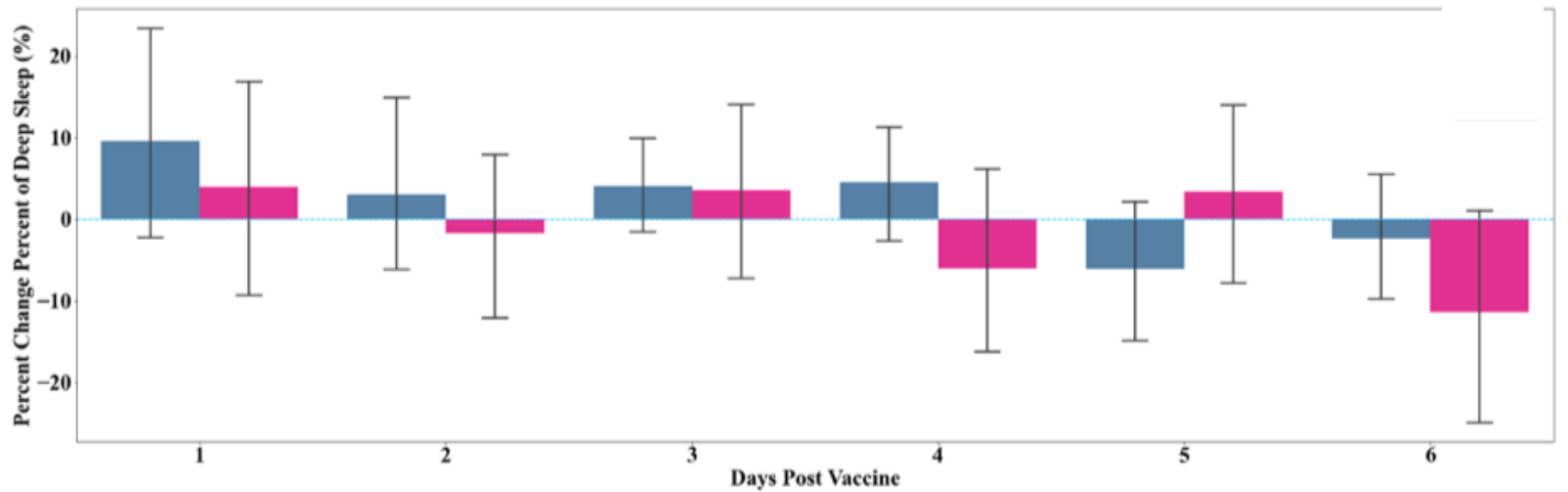

\section{Sleep Impact on HRV Changes}

Sleep duration was evaluated 7 days preceding vaccine administration to establish an individualized baseline. During the baseline assessment period, participants slept, on average, 6 hours and 43 (SD 35) minutes per night, of which $21.99 \%$ (1 hour and 28 minutes) was REM sleep and $19.1 \%$ (1 hour and 17 minutes) was deep sleep. A greater amount of sleep in the 7 days prior to receiving the first dose of the vaccine was moderately correlated with a higher percent change in HRV the 2 days following vaccine dose 1 (Pearson $R=0.570$ day 1; $R=0.494$ day 2 ).

\section{Postvaccination Symptoms}

An array of local and systemic reactions to vaccination were reported by participants, ranging from arm soreness to fatigue and body aches. A greater frequency and duration of symptoms were reported following dose 2 (Figure 4A, B). Arm soreness was reported in more than $60 \%$ of participants for both doses. The majority of symptoms subsided by hour 60 post vaccination (Figure 4A). The mean symptom duration following dose 1 was 49.7 (SD 49.2) hours, which decreased to 34.1 (SD 13.3) hours for dose 2 . The most frequent symptom duration after dose 1 and dose 2 was 24 hours. Overall, postvaccination reactogenicity would be classified as mild to moderate, as no severe adverse reactions such as angioedema or other allergic reactions requiring urgent treatment were reported $[1,20]$. Presence of postvaccination reactogenicity did not show a strong correlation with changes in sleep or other physiologic parameters. 
Figure 4. (A) Self-reported symptom duration following dose 1 and dose 2 of the COVID-19 vaccine. (B) Local and systemic reactogenicity experienced by participants by vaccine dose.
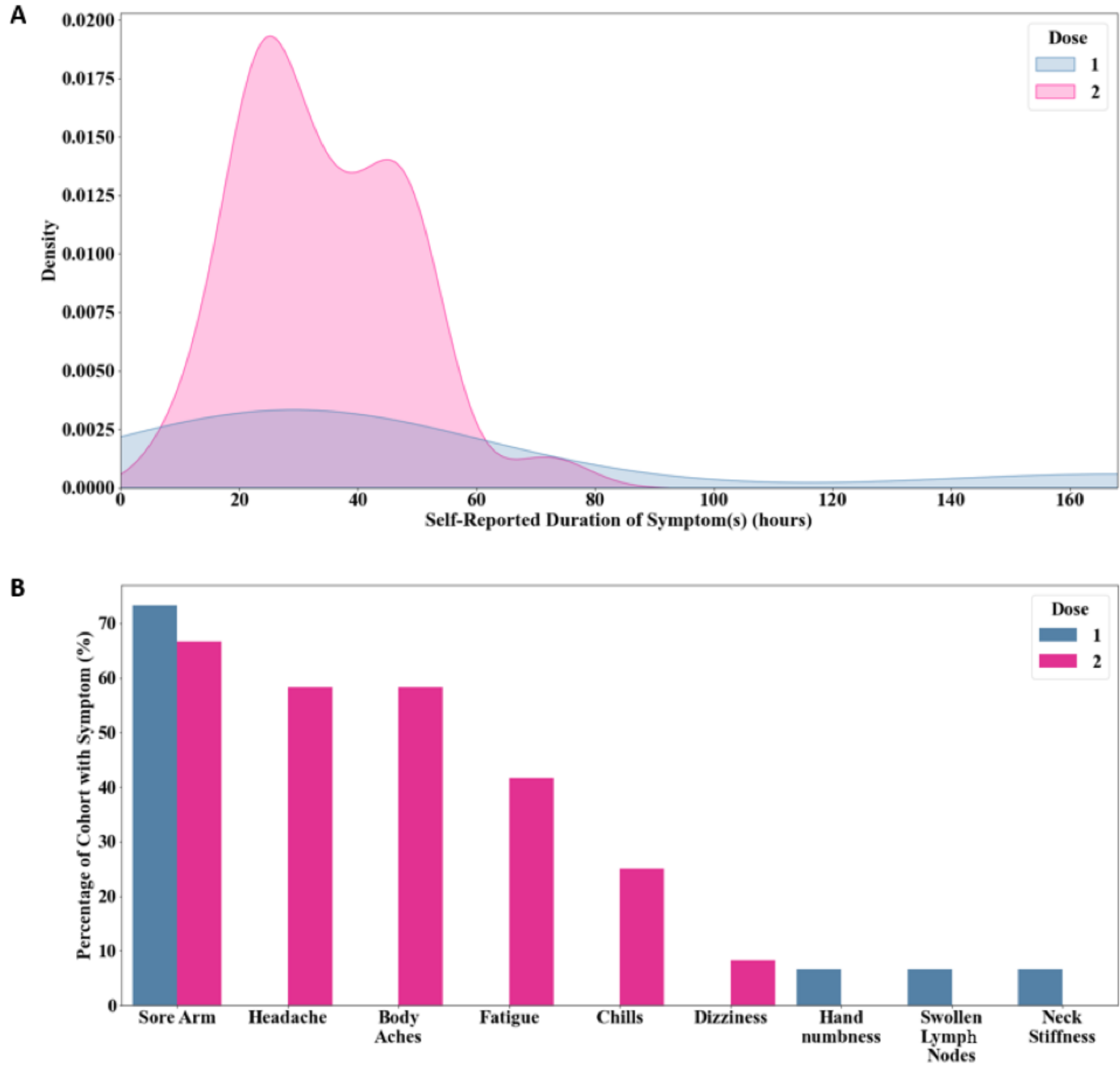

\section{Analgesic Effects on HRV, Sleep, and Postvaccination Symptoms}

None of the 19 participants premedicated with analgesic medications (ibuprofen or acetaminophen) prior to dose 1; however, 7 of the $13(54 \%)$ participants premedicated prior to dose 2 (Multimedia Appendices 4 and 5). Overall changes in HRV were the same in both groups (premedication vs no premedication; Multimedia Appendix 5). Those who did not premedicate had a greater response (increase) in RR on day 1 and day 2, but overall RR was unaffected when both groups were analyzed together (Multimedia Appendix 4). RHR had a slightly greater increase on day 1 for those who did premedicate (Multimedia Appendix 4). The group that premedicated had both a greater initial decrease and compensatory increase in total sleep duration (Multimedia Appendix 5). This group also had higher percentage of REM and deep sleep in the days after

receiving dose 2 , which were most prominent on day 1 (Multimedia Appendix 5).

The duration of all reported symptoms between the groups were similar: participants without medication experienced symptoms for a mean of 30.0 (SD 13.4) hours, and participants who self-medicated prior to dose 2 experienced symptoms for 37.7 (SD 10.0) hours. There was no significant difference in symptom severity among the two groups $[1,20]$.

\section{Discussion}

\section{Principal Findings}

In this small observational study in a relatively young and healthy cohort of participants, we provide evidence that consumer-grade wearable devices can be used to measure physiologic response to COVID-19 vaccination (Figure 1). HRV change from baseline was the most prominent signal in our 
study population, while RHR and RR were unaffected (Figure 2).

Decreases in HRV, a surrogate of autonomic tone, have been shown to predict negative clinical outcomes following severe infections [23-25]. HRV decreases have also been correlated with an increased C-reactive protein (CRP) within the first 2 days following administration of the influenza A vaccine [26]. Lower magnitude CRP elevations have been associated with increased risk of infection, suggesting that greater HRV decreases and CRP increases would equate to a protective inflammatory or immunologic response [26,27]. Thus, we propose that a higher percent change in HRV may equate to a more robust immune response to COVID-19 vaccination [14,26,28-30]. The directionality of HRV change (decrease) is significant, as this is suggestive of increased parasympathetic tone due to generation of an immune response to the vaccine [14,28]. Further investigation of HRV response to vaccination could provide a useful surrogate marker for immune system activation (Figure 5) [31]. This could be accomplished in a randomized controlled trial (RCT) of vaccination versus placebo with wearable tracking of HRV in comparison to CRP levels, antibody titers, and protection against infection.

Figure 5. Hypothetical connection between physiologic response measured by wearables (HRV, RHR, RR), inflammatory response (serum CRP and proinflammatory cytokine levels), and host immunity dictated by antibody response to vaccination. Wearable monitoring of physiologic metrics could potentially be a simple and effective way to track the efficacy of vaccine-mediated protection against infection (dashed arrow). HRV highlighted as this parameter showed the greatest changes in this study. CRP: C-reactive protein; HRV: heart rate variability; RHR: resting heart rate; RR: respiratory rate.
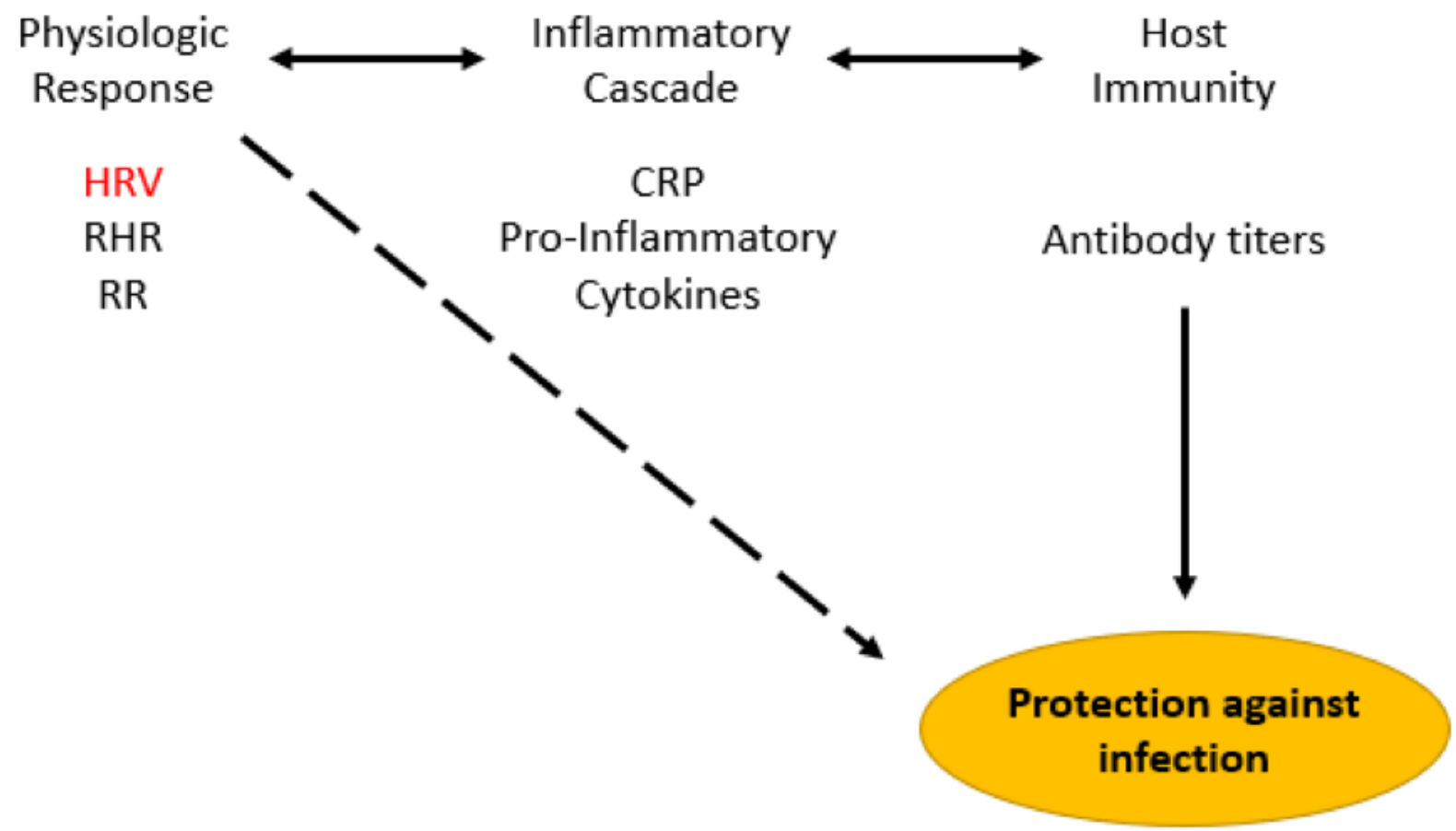

There was relatively no change in both RR and RHR in response to vaccination (Figure $2 \mathrm{~B}, \mathrm{C}$ ). This is of particular interest given that spikes in RR are clinically relevant in prediction of COVID-19 infection and progression of disease [1,11-13]. Although we did not directly collect data on oxygen saturation or hypoxia, it is likely that changes in RR are specific to COVID-19 infection, which has a predilection for pulmonary pathology. Thus, this change would not be expected with vaccination as, unlike natural disease, it does not impact pulmonary function.

Interestingly, there was a moderate correlation between change in HRV and amount of sleep prior to vaccination (greater sleep was associated with a greater decrease in HRV). Sleep deprivation is known to have a significant impact on viral susceptibility and blunted adaptive immune response in the presence of viral vaccines [32-38]. Decreased antibody titers and overall immune response have been observed in vaccinated participants that are sleep deprived, most notably in response to the influenza and hepatitis A vaccine [34-39]. A recent study of 2884 health care workers showed that 1-hour longer sleep duration was associated with $12 \%$ lower odds of COVID-19 infection [40]. Our data demonstrate that sleep duration impacts physiologic response to COVID-19 vaccination and, if correlated with immune response in further studies, could be leveraged to potentiate the effectiveness of vaccination in general.

\section{Limitations and Future Studies}

This is a small observational study in a specific cohort of participants with no control arm. A larger powered study is needed for formal statistical analysis of physiologic changes and to control for baseline demographics. The lack of a control group institutes bias; an RCT with a placebo arm (no vaccine) would allow for comparison of physiologic metrics to a control group. However, our use of percent change from baseline in our outcomes would help overcome intraindividual variability confounding of results (ie, individuals may have a greater magnitude change in parameters simply because they have a higher baseline, which is accounted for by using percent change 
from established baseline). Last, this population is known to have a greater degree of sleep deprivation secondary to duty hours and clinical demands, which may be a confounder and reduce the generalizability of the results [41].

Incorporation of biomarkers such as CRP is needed to corroborate association with physiologic changes (Figure 5) as previously observed in other vaccine studies [26,29,42,43]. Despite only exploring the response to the Pfizer-BioNTech COVID-19 vaccine, this study further confirms the feasibility of using wearable remote physiologic data to monitor responses to vaccines. This simple method to track vaccine responses would be useful for future novel vaccines. The key will be to determine how well remotely monitored physiologic metrics can predict inflammatory response, vaccination antibody titers, and ultimately protection from infection. This may provide a noninvasive method for individualized prediction of vaccine efficacy.

\section{Conclusion}

Wearable devices are now widely available to everyday consumers, and as technology has advanced, they are being more widely used to capture medical data $[7,8,12,13,44]$. This study is a proof of concept for this remote monitoring strategy to capture physiologic response to COVID-19 vaccines. If correlated with immune response and vaccine efficacy in future studies, this approach could be leveraged in the general population to predict response to vaccines. Our data also raises the possibility that increased sleep prior to vaccination may impact physiologic responses. This warrants further study and is a potentially modifiable factor to optimize vaccine response.

\section{Acknowledgments}

This study was supported by the 2020-2021 Penn State Hershey Department of Medicine House Staff Award granted to AGH and the 2020 Penn State Hershey Health Systems Science Innovation Grant awarded to AGH.

\section{Authors' Contributions}

AGH and KMD contributed equally to this study. AGH, KMD, and BB designed the research study and refined the data collection plan; BB analyzed the data with input from AGH and KMD; AGH, KMD, and BB wrote the manuscript; AKT, JBM, SB, SAM, JJC, CIP, and AT edited the manuscript.

\section{Conflicts of Interest}

CIP is a consultant for Axle Informatics for work not related to this manuscript. CIP is also the site PI for the Adaptive COVID-19 Treatment Trial (ACTT) and the ACTIV-5 / Big Effect Trial (BET-B) for the Treatment of COVID-19 which receive funding from the National Institutes of Health. This work is not related to the submitted manuscript.

\section{Multimedia Appendix 1}

Baseline characteristics of study participants.

[DOCX File, 13 KB-Multimedia Appendix 1]

\section{Multimedia Appendix 2}

Description of physiologic metrics.

[DOCX File, 112 KB-Multimedia Appendix 2]

\section{Multimedia Appendix 3}

Study flowchart. A total of 38 participants were initially enrolled in the study; 33 were screened for inclusion and exclusion criteria, yielding 19 participants that were included for final analysis (18 participants for dose 1 and 13 participants for dose 2). [PNG File, 102 KB-Multimedia Appendix 3]

\section{Multimedia Appendix 4}

Premedication subgroup analysis. Percent change from baseline in (A) heart rate variability, (B) respiratory rate, and (C) resting heart rate, measured 6 days following COVID-19 vaccine dose 2 is reported for participants who premedicated prior to vaccination (purple) and participants who did not premedicate (green). Data is reported as mean (SD).

[PNG File, 133 KB-Multimedia Appendix 4]

\section{Multimedia Appendix 5}

Premedication subgroup analysis. Percent change from baseline in (A) total sleep duration, (B) rapid eye movement sleep duration, and (C) deep sleep duration, measured 6 days following COVID-19 vaccine dose 2 is reported for participants who premedicated prior to vaccination (purple) and participants who did not premedicate (green). Data is reported as mean and SD.

[PNG File, 133 KB-Multimedia Appendix 5] 


\section{References}

1. COVID-19. Centers for Disease Control and Prevention. URL: https://www.cdc.gov/coronavirus/2019-ncov/index.html) [accessed 2003-04-21]

2. COVID-19 Dashboard by the Center for Systems Science and Engineering (CSSE) at Johns Hopkins University (JHU). Johns Hopkins Coronavirus Resource Center. URL: https://coronavirus.jhu.edu/map.html [accessed 2003-04-21]

3. Pardi N, Hogan MJ, Porter FW, Weissman D. mRNA vaccines - a new era in vaccinology. Nat Rev Drug Discov 2018 Apr;17(4):261-279 [FREE Full text] [doi: 10.1038/nrd.2017.243] [Medline: 29326426]

4. Polack FP, Thomas SJ, Kitchin N, Absalon J, Gurtman A, Lockhart S, C4591001 Clinical Trial Group. Safety and efficacy of the BNT162b2 mRNA Covid-19 vaccine. N Engl J Med 2020 Dec 31;383(27):2603-2615 [FREE Full text] [doi: 10.1056/NEJMoa2034577] [Medline: 33301246 ]

5. Sahin U, Muik A, Vogler I, Derhovanessian E, Kranz LM, Vormehr M, et al. BNT162b2 induces SARS-CoV-2-neutralising antibodies and T cells in humans. medRxiv. Preprint posted online on December 11, 2020.

6. Wang Z, Schmidt F, Weisblum Y, Muecksch F, Barnes CO, Finkin S, et al. mRNA vaccine-elicited antibodies to SARS-CoV-2 and circulating variants. Nature 2021 Apr;592(7855):616-622. [doi: 10.1038/s41586-021-03324-6] [Medline: 33567448]

7. Vogels EA. About one-in-five Americans use a smart watch or fitness tracker. Pew Research Center. 2020. URL: https:/ /www.pewresearch.org/fact-tank/2020/01/09/about-one-in-five-americans-use-a-smart-watch-or-fitness-tracker/ [accessed 2021-03-04]

8. Greiwe J, Nyenhuis SM. Wearable technology and how this can be implemented into clinical practice. Curr Allergy Asthma Rep 2020 Jun 06;20(8):36 [FREE Full text] [doi: 10.1007/s11882-020-00927-3] [Medline: 32506184]

9. Berryhill S, Morton CJ, Dean A, Berryhill A, Provencio-Dean N, Patel SI, et al. Effect of wearables on sleep in healthy individuals: a randomized crossover trial and validation study. J Clin Sleep Med 2020 May 15;16(5):775-783. [doi: 10.5664/jcsm.8356] [Medline: 32043961]

10. Stein PK, Bosner MS, Kleiger RE, Conger BM. Heart rate variability: a measure of cardiac autonomic tone. Am Heart J 1994 May;127(5):1376-1381. [doi: 10.1016/0002-8703(94)90059-0] [Medline: 8172068]

11. Miller DJ, Capodilupo JV, Lastella M, Sargent C, Roach GD, Lee VH, et al. Analyzing changes in respiratory rate to predict the risk of COVID-19 infection. PLoS One 2020;15(12):e0243693 [FREE Full text] [doi: 10.1371/journal.pone.0243693] [Medline: 33301493 ]

12. Mishra T, Wang M, Metwally AA, Bogu GK, Brooks AW, Bahmani A, et al. Pre-symptomatic detection of COVID-19 from smartwatch data. Nat Biomed Eng 2020 Dec;4(12):1208-1220. [doi: 10.1038/s41551-020-00640-6] [Medline: 33208926]

13. Hirten RP, Danieletto M, Tomalin L, Choi KH, Zweig M, Golden E, et al. Use of physiological data from a wearable device to identify SARS-CoV-2 infection and symptoms and predict COVID-19 diagnosis: observational study. J Med Internet Res 2021 Feb 22;23(2):e26107 [FREE Full text] [doi: 10.2196/26107] [Medline: 33529156]

14. Hasty F, García G, Dávila CH, Wittels SH, Hendricks S, Chong S. Heart rate variability as a possible predictive marker for acute inflammatory response in COVID-19 patients. Mil Med 2020 Nov 18:e34 [FREE Full text] [doi:

10.1093/milmed/usaa405] [Medline: $\underline{33206183}$ ]

15. Remote physiologic monitoring of resident wellness and burnout. ClinicalTrials.gov. 2020. URL: https://clinicaltrials.gov/ ct2/show/NCT04304703 [accessed 2021-03-04]

16. Shaffer F, Ginsberg JP. An overview of heart rate variability metrics and norms. Front Public Health 2017;5:258. [doi: 10.3389/fpubh.2017.00258] [Medline: 29034226]

17. Herzig D, Eser P, Omlin X, Riener R, Wilhelm M, Achermann P. Reproducibility of heart rate variability is parameter and sleep stage dependent. Front Physiol 2017;8:1100. [doi: 10.3389/fphys.2017.01100] [Medline: 29367845]

18. Yasuma F, Hayano J. Respiratory sinus arrhythmia: why does the heartbeat synchronize with respiratory rhythm? Chest 2004 Mar;125(2):683-690. [doi: 10.1378/chest.125.2.683] [Medline: 14769752]

19. Silvani A, Dampney RAL. Central control of cardiovascular function during sleep. Am J Physiol Heart Circ Physiol 2013 Dec;305(12):H1683-H1692 [FREE Full text] [doi: 10.1152/ajpheart.00554.2013] [Medline: 24097430]

20. Local reactions, systemic reactions, adverse events, and serious adverse events: Pfizer-BioNTech COVID-19 vaccine. Centers for Disease Control and Prevention. URL: https://www.cdc.gov/vaccines/covid-19/info-by-product/pfizer/ reactogenicity.html?CDC_AA_refVal=https $\% 3 \mathrm{~A} \% 2 \mathrm{~F} \% 2 \mathrm{Fwww} . \mathrm{cdc}$. gov\%2Fvaccines\%2Fcovid-19\%2Finfo-by-manufacturer\%2Fpfizer\%2Freactogenicity.html [accessed 2021-03-04]

21. Pearson correlation coefficient and p-value for testing non-correlation. SciPy. URL: https://docs.scipy.org/doc/scipy/ reference/generated/scipy.stats.pearsonr.html [accessed 2021-02-27]

22. Plot univariate or bivariate distributions using kernel density estimation. seaborn. URL: https://seaborn.pydata.org/generated/ seaborn.kdeplot.html [accessed 2021-02-27]

23. Ahmad S, Ramsay T, Huebsch L, Flanagan S, McDiarmid S, Batkin I, et al. Continuous multi-parameter heart rate variability analysis heralds onset of sepsis in adults. PLoS One 2009 Aug 14;4(8):e6642 [FREE Full text] [doi:

10.1371/journal.pone.0006642] [Medline: 19680545] 
24. Kovatchev BP, Farhy LS, Cao H, Griffin MP, Lake DE, Moorman JR. Sample asymmetry analysis of heart rate characteristics with application to neonatal sepsis and systemic inflammatory response syndrome. Pediatr Res 2003 Dec;54(6):892-898. [doi: 10.1203/01.PDR.0000088074.97781.4F] [Medline: 12930915]

25. de Castilho FM, Ribeiro ALP, Nobre V, Barros G, de Sousa MR. Heart rate variability as predictor of mortality in sepsis: a systematic review. PLoS One 2018;13(9):e0203487 [FREE Full text] [doi: 10.1371/journal.pone.0203487] [Medline: $\underline{30204803}$

26. Lanza GA, Barone L, Scalone G, Pitocco D, Sgueglia GA, Mollo R, et al. Inflammation-related effects of adjuvant influenza A vaccination on platelet activation and cardiac autonomic function. J Intern Med 2011 Jan;269(1):118-125. [doi: 10.1111/j.1365-2796.2010.02285.x] [Medline: 20964738]

27. McDade TW, Borja JB, Kuzawa CW, Perez TLL, Adair LS. C-reactive protein response to influenza vaccination as a model of mild inflammatory stimulation in the Philippines. Vaccine 2015 Apr 21;33(17):2004-2008 [FREE Full text] [doi: 10.1016/j.vaccine.2015.03.019] [Medline: 25795257]

28. Perring S, Jones E. Assessment of changes in cardiac autonomic tone resulting from inflammatory response to the influenza vaccination. Clin Physiol Funct Imaging 2012 Nov;32(6):437-444. [doi: 10.1111/j.1475-097X.2012.01147.x] [Medline: 23031064]

29. Tsai MY, Hanson NQ, Straka RJ, Hoke TR, Ordovas JM, Peacock JM, et al. Effect of influenza vaccine on markers of inflammation and lipid profile. J Lab Clin Med 2005 Jun;145(6):323-327. [doi: 10.1016/j.lab.2005.03.009] [Medline: 15976761]

30. Young HA, Benton D. Heart-rate variability: a biomarker to study the influence of nutrition on physiological and psychological health? Behav Pharmacol 2018 Apr;29(2 and 3-Spec Issue):140-151 [FREE Full text] [doi: 10.1097/FBP.0000000000000383] [Medline: 29543648]

31. Williams DP, Koenig J, Carnevali L, Sgoifo A, Jarczok MN, Sternberg EM, et al. Heart rate variability and inflammation: a meta-analysis of human studies. Brain Behav Immun 2019 Aug;80:219-226. [doi: 10.1016/j.bbi.2019.03.009] [Medline: 30872091]

32. Prather AA, Janicki-Deverts D, Hall MH, Cohen S. Behaviorally assessed sleep and susceptibility to the common cold. Sleep 2015 Sep 01;38(9):1353-1359 [FREE Full text] [doi: 10.5665/sleep.4968] [Medline: 26118561]

33. Spiegel K, Sheridan JF, Van Cauter E. Effect of sleep deprivation on response to immunization. JAMA 2002 Sep 25;288(12):1471-1472. [doi: 10.1001/jama.288.12.1471-a] [Medline: 12243633]

34. Taylor DJ, Kelly K, Kohut ML, Song K. Is insomnia a risk factor for decreased influenza vaccine response? Behav Sleep Med 2017;15(4):270-287 [FREE Full text] [doi: 10.1080/15402002.2015.1126596] [Medline: 27077395]

35. Lange T, Perras B, Fehm HL, Born J. Sleep enhances the human antibody response to hepatitis A vaccination. Psychosom Med 2003;65(5):831-835. [doi: 10.1097/01.psy.0000091382.61178.f1] [Medline: 14508028]

36. Prather AA, Pressman SD, Miller GE, Cohen S. Temporal links between self-reported sleep and antibody responses to the influenza vaccine. Int J Behav Med 2021 Mar;28(1):151-158. [doi: 10.1007/s12529-020-09879-4] [Medline: 32236831]

37. Benedict C, Brytting M, Markström A, Broman J, Schiöth HB. Acute sleep deprivation has no lasting effects on the human antibody titer response following a novel influenza A H1N1 virus vaccination. BMC Immunol 2012 Jan 04;13:1 [FREE Full text] [doi: 10.1186/1471-2172-13-1] [Medline: 22217111]

38. Prather AA, Hall M, Fury JM, Ross DC, Muldoon MF, Cohen S, et al. Sleep and antibody response to hepatitis B vaccination. Sleep 2012 Aug 01;35(8):1063-1069 [FREE Full text] [doi: 10.5665/sleep.1990] [Medline: 22851802]

39. Haspel JA, Anafi R, Brown MK, Cermakian N, Depner C, Desplats P, et al. Perfect timing: circadian rhythms, sleep, and immunity - an NIH workshop summary. JCI Insight 2020 Jan 16;5(1):e131487. [doi: 10.1172/jci.insight.131487] [Medline: $\underline{31941836}$ ]

40. Kim H, Hegde S, LaFiura C, Raghavan M, Luong E, Cheng S, et al. COVID-19 illness in relation to sleep and burnout. BMJ Nutr Prev Health 2021;4(1):132-139 [FREE Full text] [doi: 10.1136/bmjnph-2021-000228] [Medline: 34308120 ]

41. Morales J, Yáñez A, Fernández-González L, Montesinos-Magraner L, Marco-Ahulló A, Solana-Tramunt M, et al. Stress and autonomic response to sleep deprivation in medical residents: a comparative cross-sectional study. PLoS One 2019;14(4):e0214858 [FREE Full text] [doi: 10.1371/journal.pone.0214858] [Medline: 30947295]

42. Christian LM, Porter K, Karlsson E, Schultz-Cherry S. Proinflammatory cytokine responses correspond with subjective side effects after influenza virus vaccination. Vaccine 2015 Jun 26;33(29):3360-3366 [FREE Full text] [doi: 10.1016/j.vaccine.2015.05.008] [Medline: 26027906]

43. Whelton SP, Narla V, Blaha MJ, Nasir K, Blumenthal RS, Jenny NS, et al. Association between resting heart rate and inflammatory biomarkers (high-sensitivity C-reactive protein, interleukin-6, and fibrinogen) (from the Multi-Ethnic Study of Atherosclerosis). Am J Cardiol 2014 Mar 15;113(4):644-649 [FREE Full text] [doi: 10.1016/j.amjcard.2013.11.009] [Medline: 24393259]

44. Guk K, Han G, Lim J, Jeong K, Kang T, Lim E, et al. Evolution of wearable devices with real-time disease monitoring for personalized healthcare. Nanomaterials (Basel) 2019 May 29;9(6):813 [FREE Full text] [doi: 10.3390/nano9060813] [Medline: $\underline{31146479}$ ] 


\author{
Abbreviations \\ CRP: C-reactive protein \\ HRV: heart rate variability \\ mRNA: messenger RNA \\ RCT: randomized controlled trial \\ REM: rapid eye movement \\ RHR: resting heart rate \\ rpm: respirations per minute \\ RR: respiratory rate
}

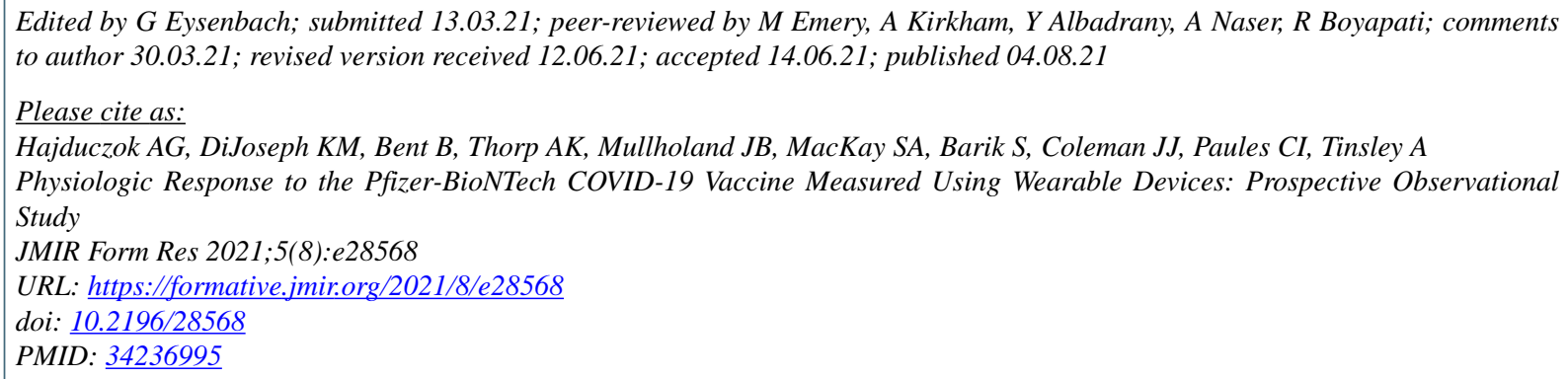

(OAlexander G Hajduczok, Kara M DiJoseph, Brinnae Bent, Audrey K Thorp, Jon B Mullholand, Stuart A MacKay, Sabrina Barik, Jamie J Coleman, Catharine I Paules, Andrew Tinsley. Originally published in JMIR Formative Research (https://formative.jmir.org), 04.08.2021. This is an open-access article distributed under the terms of the Creative Commons Attribution License (https://creativecommons.org/licenses/by/4.0/), which permits unrestricted use, distribution, and reproduction in any medium, provided the original work, first published in JMIR Formative Research, is properly cited. The complete bibliographic information, a link to the original publication on https://formative.jmir.org, as well as this copyright and license information must be included. 